\title{
Parâmetros genéticos e tendências genética e fenotípica para características de crescimento em uma população da raça Brangus
}

\section{Jader Silva Lopes ${ }^{1}$, Paulo Roberto Nogara Rorato ${ }^{2}$, Tomás Weber ${ }^{1}$, Mariana de Almeida Dornelles $^{3}$, Juliana Grigoletto Comin ${ }^{3}$, Ronyere Olegário de Araújo ${ }^{1}$}

\author{
${ }^{1}$ Curso de Pós-Graduação em Zootecnia da UFSM. Bolsista CAPES. \\ 2 Departamento de Zootecnia da UFSM. \\ ${ }^{3}$ Curso de Graduação em Zootecnia da UFSM.
}

\begin{abstract}
RESUMO - Para estimar parâmetros genéticos e a tendência genética e fenotípica para peso à desmama (PD), peso ao sobreano (PS), ganho médio diário do nascimento à desmama (GMDND), ganho médio diário da desmama ao sobreano (GMDDS), dias para atingir $160 \mathrm{~kg}$ na fase pré-desmama (D160) e dias para atingir $240 \mathrm{~kg}$ na fase pós-desmama (D240), foram utilizados os registros de desempenho de 9.573 animais da raça Brangus, filhos de 415 touros e 7.297 vacas, criados em 37 propriedades, nas regiões Sul, Sudeste e Centro-Oeste do Brasil entre os anos de 1987 e 2001. Os componentes de co-variância foram obtidos pelo REML, utilizando-se um modelo animal incluindo, como fixos, os efeitos de grupo genético do pai e da mãe e de grupo de contemporâneos, além das co-variáveis idade da vaca ao parto, idade à pesagem à desmama e idade à pesagem ao sobreano, lineares e quadráticas, e, como aleatórios, os efeitos genéticos aditivos diretos e maternos e o residual. As médias observadas foram: 171,62 kg para PD; 306,82 kg para PS; 0,701 kg para GMDND; 0,395 kg para GMDDS; 234,97 dias para D160; 720,52 dias para D240. As herdabilidades diretas variaram de 0,15 (D240) a 0,45 (GMDND) e as maternas, de $0,20(\mathrm{PD})$ a 0,28 (GMDND). As tendências genéticas e fenotípicas foram de 7,59 g/ano e 0,864 kg/ano para peso à desmama; $5,16 \mathrm{~g} / \mathrm{ano}$ e $2,274 \mathrm{~kg} /$ ano para peso ao sobreano; $0,036 \mathrm{~g} / \mathrm{ano}$ e $0,007 \mathrm{~kg} / \mathrm{ano}$ para ganho médio diário do nascimento à desmama; 0,011 g/ano e 0,012 kg/ano para ganho médio diário da desmama ao sobreano; -0,11 dias/ano e -1,59 dias/ano para dias para atingir $160 \mathrm{~kg}$ na fase pré-desmama; -0,78 dias/ano e -9,79 dias/ano para atingir $240 \mathrm{~kg}$ na fase pós-desmama.
\end{abstract}

Palavras-chave: herdabilidade, máxima verossimilhança restrita, valor genético

\section{Genetic parameters and genetic and phenotypic trends for growing traits for a Brangus breed population}

\begin{abstract}
This study aimed to estimate the genetic parameters and genetic and phenotypic trends for weight at weaning (PD), yearling weight (PS), average daily gain from birth to weaning (GMDND), average daily gain from weaning to yearling (GMDDS), days to reach $160 \mathrm{~kg}$ at the pre weaning phase (D160) and days to reach $240 \mathrm{~kg}$ at post weaning phase (D240) of Brangus cattle raised in 37 farms in South, Southern and Mid Western Regions of Brazil, from 1987 to 2001. Records on 9,573 Brangus animals, sired by 415 bulls and 7,297 dams, were used to estimate (co)variance components by REML.The model included the genetic group of bull and dam and of contemporary group, age of cow at deliver, age at weaning weight and age at yearling weight covariate (linear and quadratic componentes) as fixed effects and the direct additive genetic, maternal and residual as random effects. The averages observed for PD, PS, GMDND, GMDDS, D160 and D240 were $171.62 \mathrm{~kg}, 306.2 \mathrm{~kg}, 0.701 \mathrm{~kg}, 0.395 \mathrm{~kg}, 234.97$ days and 720.52 days, respectively. The direct heritabilities ranged from 0.15 (D240) to 0.45 (GMDND) and the maternal from 0.20 (PD) to 0.28 (GMDND). The genetic and phenotypic trends were $7.59 \mathrm{~g} / \mathrm{year}$ and $0.864 \mathrm{~kg} / \mathrm{year} ; 5.16 \mathrm{~g} / \mathrm{year}$ and $2.274 \mathrm{~kg} / \mathrm{year} ; 0.036 \mathrm{~g} / \mathrm{year}$ and $0.007 \mathrm{~kg} /$ year; $0.011 \mathrm{~g} / \mathrm{year}$ and $0.012 \mathrm{~kg} / \mathrm{year}$; -0.11 days/year and -1.59 days/year; -0.78 days/year and -9.79 days/year, respectively, for PD, PS, GMDND, GMDDS, D160 and D240.
\end{abstract}

Key Words: breeding value, heritability, Restricted Maximum Likelihood

\section{Introdução}

A cadeia produtiva de carne bovina tem se tornado mais competitiva e as margens de lucro têm diminuído, levando produtores à busca por tecnologias disponíveis visando ao aumento da produtividade. Nesse sentido, o conhecimento das potencialidades e limitações das raças é importante, pois fornece orientação para programas de cruzamento 
voltados ao aproveitamento dos benefícios da heterose, da combinação aditiva e da complementaridade entre as raças com o objetivo final de melhorar a eficiência e a qualidade do produto. O cruzamento entre raças européias e zebuínas tem sido utilizado amplamente nos sistemas de criação brasileiros com o objetivo de produzir animais com maior desempenho nas condições de ambiente em que são explorados.

É fundamental que, paralelamente à evolução fenotípica de uma população, seja em raça pura ou produto de cruzamento, esta população evolua também geneticamente, caso contrário, o avanço fenotípico será limitado. Desta forma, se não houver progresso genético, não ocorrerá progresso fenotípico (Fernandes et al., 2002).

O melhoramento genético é um dos principais meios para se obter evolução fenotípica em um rebanho, porém, uma vez implantado, é necessária a verificação periódica de sua eficiência (Mello et al., 2002), ou seja, se o progresso genético esperado está ocorrendo. O progresso genético é medido pelo cálculo da tendência genética da população, que determina quanto da variação anual do rebanho tem origem genética. Para isso, é necessário que a mudança fenotípica seja decomposta nos seus componentes genéticos e de ambiente (Oliveira et al., 1995).

Procedimentos de modelos mistos podem ser usados para analisar dados e produzir estimativas da variância genética e da resposta à seleção em uma única análise (Sorensen \& Kennedy, 1986). A metodologia de modelos mistos para o modelo animal incorpora a matriz das relações genéticas aditivas, considerando todas as co-variâncias (tanto aquelas entre indivíduos dentro de uma geração como aquelas entre indivíduos de diferentes gerações). Para estimar a resposta à seleção, considerando o fato de que as famílias fornecem número de informações diferentes, esta metodologia pondera corretamente os indivíduos de acordo com registros da geração corrente e de todas as gerações passadas. A omissão do relacionamento entre gerações tem implicações importantes na análise de dados gerados por seleção (Sorensen \& Kennedy, 1984; 1986), uma vez que experimentos de seleção são processos estocásticos e o desempenho em determinada geração depende da amostra genética retida na geração anterior (Hill, 1980).

O objetivo neste estudo foi estimar os coeficientes de herdabilidade e as tendências genéticas e fenotípicas para as características peso à desmama e ao sobreano, ganho médio de peso do nascimento à desmama e da desmama ao sobreano e dias para atingir 160 e $240 \mathrm{~kg}$ de peso vivo em uma população da raça Brangus.

\section{Material e Métodos}

O banco de dados utilizado neste estudo continha registros de desempenho de 9.573 animais da raça Brangus, filhos de 415 touros e 7.297 vacas, criados em 37 fazendas localizadas nas regiões Sul, Sudeste e Centro-Oeste do Brasil, coletados no período entre 1987 e 2001 e cedido por Gensys Consultores Associados S/C Ltda. e Natura Genética Sul-Americana.

$\mathrm{Na}$ montagem do arquivo de trabalho, foram geradas, usando o aplicativo SAS (2001), as seguintes variáveis: grupo de contemporâneos (GC), agrupando os animais nascidos na mesma fazenda, no mesmo ano, na mesma estação (meses de janeiro a março $=1$, de abril a junho $=2$, de julho a setembro $=3$ e de outubro a dezembro $=4$ ) e pertencentes ao mesmo sexo e grupo de manejo; grupo genético dos pais (GG), formado pelo numerador da fração Angus (A) Nelore (N) do pai e da mãe do produto, totalizando quatro grupos diferentes (Tabela 1); ganho de peso médio diário do nascimento à desmama (GMDND); ganho de peso médio diário da desmama ao sobreano (GMDDS); dias para atingir $160 \mathrm{~kg}$ na fase pré-desmama (D160) e dias para atingir $240 \mathrm{~kg}$ na fase pós-desmama (D240).

Foram excluídos do arquivo de trabalho os grupos de contemporâneos com menos de cinco animais; os reprodutores com menos de cinco filhos; os registros incompletos e os animais com desempenho fora do intervalo de 2,5 desvios-padrão, para mais ou para menos, em relação à média da população.

Os componentes de co-variância utilizados para estimar os coeficientes de herdabilidade e predizer os valores genéticos foram obtidos pelo Método da Máxima Verossimilhança Restrita livre de derivada, por meio do programa MTDFREML, descrito por Boldman et al. (2001). Foi adotado um modelo animal considerando o peso à desmama (PD), o ganho médio diário do nascimento à desmama (GMDND) e o número de dias para atingir $160 \mathrm{~kg}$ na fase pré-desmama (D160) em função dos efeitos fixos de grupo genético, do grupo de contemporâneos na pré-desmama, além das co-variáveis idade da vaca ao parto (IVP) e idade à pesagem à desmama (IPD), lineares e quadráticas, e dos efeitos aleatórios, genéticos aditivos diretos e maternos e o residual. Para peso ao sobreano (PS), GMDDS e D240, o modelo adotado foi similar, excluindo a co-variável IVP e os efeitos aditivos maternos e incluindo o grupo de contemporâneos na pós-desmama e a idade à pesagem ao sobreano (IPS), em substituição a grupo de contemporâneos na pré-desmama e idade à pesagem à desmama. As idades médias dos animais foram 
Tabela 1 - Distribuição dos produtos Brangus em cada grupo genético dos pais

\begin{tabular}{|c|c|c|c|c|}
\hline \multirow[t]{2}{*}{ Grupo genético } & \multirow[t]{2}{*}{ Número de animais } & \multicolumn{3}{|c|}{ Composição racial } \\
\hline & & Pai & Mãe & Produto \\
\hline 1310 & 1.812 & $1 / 4 \mathrm{~A} \times 3 / 4 \mathrm{~N}$ & $1 \mathrm{~A} \times 0 \mathrm{~N}$ & $5 / 8 \mathrm{~A} \times 3 / 8 \mathrm{~N}$ \\
\hline 5353 & 3.591 & $5 / 8 \mathrm{~A} \times 3 / 8 \mathrm{~N}$ & $5 / 8 \mathrm{~A} \times 3 / 8 \mathrm{~N}$ & $5 / 8 \mathrm{~A} \times 3 / 8 \mathrm{~N}$ \\
\hline 3111 & 1.369 & $3 / 4 \mathrm{~A} \times 1 / 4 \mathrm{~N}$ & $1 / 2 \mathrm{~A} \times 1 / 2 \mathrm{~N}$ & $5 / 8 \mathrm{~A} \times 3 / 8 \mathrm{~N}$ \\
\hline 1013 & 2.801 & $1 \mathrm{~A} \times 0 \mathrm{~N}$ & $1 / 4 \mathrm{~A} \times 3 / 4 \mathrm{~N}$ & $5 / 8 \mathrm{~A} \times 3 / 8 \mathrm{~N}$ \\
\hline
\end{tabular}

196, 531 e 1.992 dias, respectivamente, para idade à pesagem à desmama, IPS e IVP.

O modelo de análise utilizado sob a forma matricial foi o seguinte:

$$
\mathrm{y}=\mathrm{X} \beta+\mathrm{Z}_{1} \mathrm{a}+\mathrm{Z}_{2} \mathrm{~m}+\mathrm{e},
$$

em que: $\mathrm{y}=$ vetor das observações estudadas (PD, PS, GMDND, GMDDS, D160 e D240); X = matriz de incidência associada aos efeitos fixos; $\beta=$ vetor de solução para os efeitos fixos; $Z_{1}=$ matriz de incidência associada ao efeito genético direto; $\mathrm{a}=$ vetor de soluções para o efeito genético direto; $\mathrm{Z}_{2}=$ matriz de incidência associada ao efeito genético materno; $m$ = vetor de soluções para o efeito genético materno; e = vetor dos resíduos, aleatórios.

As pressuposições assumidas para os vetores $y, a, m$ e $e$ nas análises bi-variadas foram: $\mathrm{E}[\mathrm{y}]=\mathrm{X} \beta ; \operatorname{Var}(\mathrm{a})=$ $A \otimes G_{a}, \operatorname{Var}(m)=A \otimes G_{m}, \operatorname{Var}(e)=I \otimes R_{e}$, em que $\mathrm{G}_{\mathrm{a}}=$ matriz de variâncias e co-variâncias genéticas aditivas; $\mathrm{G}_{\mathrm{m}}=$ matriz de variâncias e co-variâncias genéticas maternas; $\mathrm{R}_{\mathrm{e}}=$ matriz de variância e co-variância residual; $\mathrm{A}=$ matriz das relações genético-aditivas; $\mathrm{I}=$ matriz identidade; e $\otimes=$ produto direto entre matrizes. Assumiu-se ainda que os vetores $a, m$ e $e$ são não correlacionados entre si.

O critério de convergência considerado nas análises com o MTDFREML foi $10^{-6}$, de modo que, a cada convergência, o programa foi reiniciado usando como valores iniciais aqueles obtidos na análise anterior. A matriz de parentesco foi constituída de 15.759 animais.

No estudo da tendência genética, utilizou-se a regressão dos valores genéticos preditos, com base nas médias ponderadas pelo número de observações, dentro de seu respectivo ano de nascimento, obtidas pelo procedimento PROC REG do pacote estatístico SAS (2001), utilizando um modelo estatístico do tipo: $\mathrm{Y}_{\mathrm{ij}}=\mathrm{b}_{\mathrm{o}}+\mathrm{b}_{1} \mathrm{x}_{\mathrm{i}}$ $+\mathrm{e}_{\mathrm{ij}}$, em que: $\mathrm{Y}_{\mathrm{ij}}=$ valor genético $(\mathrm{VG})$ para as características em estudo (P205, P550, GMDND, GMDDS, D160 e D240) do animal $\mathrm{j}$ nascido no ano $\mathrm{i} ; \mathrm{b}_{\mathrm{o}}=$ intercepto; $\mathrm{b}_{1}=$ coeficiente angular da reta; $\mathrm{x}_{\mathrm{i}}=$ ano de nascimento $\mathrm{i} ; \mathrm{e}_{\mathrm{ij}}=$ erro aleatório.

\section{Resultados e Discussão}

O número de animais controlados por ano tendeu a aumentar no decorrer do período estudado (Figura 1), o que sugere uma preocupação crescente dos criadores em conhecer geneticamente seus animais ou, ainda, crescimento no uso da raça Brangus neste período.

As médias fenotípicas para peso à desmama e peso ao sobreano foram 171,62 e $306,82 \mathrm{~kg}$, respectivamente, superiores às relatadas por Souza et al. (2007), PD = 162,3 kg, para a raça Angus e por Guterres et al. (2007), PS =292,95 kg para a raça Brangus. Todavia, a média de peso à desmama foi inferior à encontrada por Guterres et al. (2007), PD = $173,35 \mathrm{~kg}$. Para GMDND e GMDDS, as médias fenotípicas foram 701 e $395 \mathrm{~g} / \mathrm{dia}$, superiores às relatadas por Guterres et al. (2007), de 699,29 e 346,39 g/dia, e por Balbé et al. (2007) para GMDDS na raça Brangus, 384,22 g/dia.

As médias fenotípicas para D160 e D240 foram 234,97 e 720,52 dias, respectivamente; a primeira superior e a segunda próxima aos valores relatados por Malhado et al. (2005), de 265 dias para D160 e 718 dias para D240 para a raça Nelore, o que mostra maior velocidade de crescimento na fase prédesmama para a raça Brangus. Considerando que o ideal seria produzir um bezerro com $190 \mathrm{~kg}$ aos 205 dias de idade

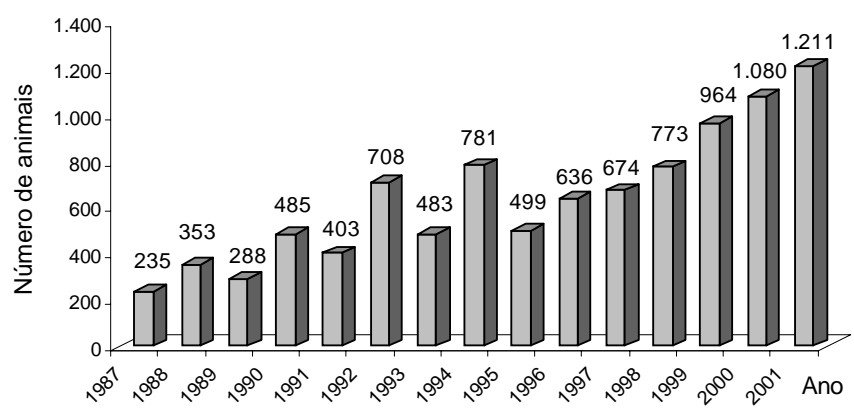

Figura 1 - Número de animais controlados por ano de nascimento em uma população da raça Brangus. 
e peso de abate de $450 \mathrm{~kg}$ aos 24 meses (Malhado et al., 2005), os resultados deste trabalho indicam que a população estudada está próxima desses valores para a prédesmama, pois atingiriam aproximadamente $183 \mathrm{~kg}$ aos 205 dias, enquanto o desempenho pós-desmama está aquém do desejado, pois os animais atingiriam o peso de abate somente aos 2 anos e meio de idade.

As herdabilidades diretas (Tabela 2) variaram de 0,15 (D240) a 0,45 (GMDND) e indicam que o valor fenotípico pode ser utilizado como indicador do valor genético aditivo direto e o uso da seleção possibilita ganho genético de baixo a moderado nível para estas características. Valores semelhantes foram relatados por Mercadante \& Lobo (1998) para pós-desmama (0,29); por Euclides Filho et al. (2000) para ganho de peso dos 12 aos 18 meses (0,34); por Marcondes et al. (2000) para GMDND $(0,18)$; Marcondes et al. (2002) para peso à desmama $(0,23)$ e para peso ao sobreano (0,30); e por Sarmento et al. (2003) para ganho médio diário dos 365 aos 550 dias de idade $(0,21)$, todos na raça Nelore; além de Balbé et al. (2007) para GMDDS $(0,30)$, para a raça Brangus. Entretanto, são superiores aos relatados por Holanda et al. (2004) para GMDND na raça Nelore $(0,06)$ e inferiores ao estimado por Souza et al. (2007) para peso à desmama $(0,56)$ na raça Angus.

As estimativas de herdabilidade materna (Tabela 2) encontradas foram similares ao valor relatado por Balbé et al. (2007) para GMDDS $(0,29)$ e superiores às encontradas por Eler et al. (1995), 0,13 para peso à desmama; por Garnero et al. (1998), de 0,00 a 0,16 para pesos aos 120 e 550 dias de idade; por Mercadante \& Lobo (1998), de 0,13 para peso à desmama e 0,09 para peso aos 365 dias de idade; e Marcondes et al. (2002), PS =0,03, todos para a raça Nelore. Segundo Garnero et al. (1998), esses baixos valores estão relacionados ao antagonismo entre os efeitos dos genes para potencial de crescimento e aqueles para habilidade materna. Os valores estimados para as herdabilidades maternas neste trabalho (de 0,20 a 0,28) indicam que é possível obter ganho genético por meio de seleção para esta característica.

As correlações entre os efeitos genéticos diretos e maternos foram iguais a 0,55 para peso à desmama; 0,45 para GMDND e 0,33 para D160, o que indica sinergismo entre os efeitos dos genes que determinam o potencial de crescimento do bezerro e aqueles responsáveis pela habilidade materna. Correlação alta e positiva entre os efeitos direto e materno também foi relatada por Marques et al. (2000) para peso aos 100 dias de idade $(0,85)$. Entretanto, os resultados obtidos neste trabalho diferem das correlações genéticas negativas relatadas por Sarmento et al. (2003) para as características de ganho de peso $(-0,77 ;-0,24 ; \mathrm{e}-0,44$, respectivamente, para GMDND, GMDDAno e GMDDS) e por Souza et al. (2007) para peso à desmama $(-0,83)$.

As estimativas de correlação genética estimadas entre as características estudadas variaram de 0,03 (GMDND $\times$ GMDDS ) a 0,50 (PD × PS), o que indica, no primeiro caso, que a seleção para uma das características não promove ganho na outra e, no segundo caso, que a seleção para uma promoverá ganho na outra.

As tendências genéticas diretas anuais (Figura 2) para as características pré-desmama não foram significativas $(\mathrm{P}<0,10)$. Para peso à desmama e GMDND, os valores destas estimativas foram próximos de zero, portanto, as mudanças genéticas anuais não foram suficientes para considerar que esta população esteja evoluindo geneticamente e que maior atenção deve ser dada à seleção dos indivíduos a serem utilizados para a reprodução. Entretanto, as tendências direta e materna para D160 foram -0,11 e -0,25 dias/ano, respectivamente, assim, em termos de mudança genética direta anual, 0,05\% da média fenotípica equivale a uma redução igual a 1,65 (direta) e 3,67 (materna) dias para ganhar $160 \mathrm{~kg}$, na pré-desmama nos 15 anos avaliados e provavelmente esteve relacionada à habilidade materna superior, característica na raça Angus,

Tabela 2 - Coeficientes de herdabilidade direta (d) e materna (m), diagonal principal, e das correlações genéticas, abaixo da diagonal, para uma população da raça Brangus

\begin{tabular}{|c|c|c|c|c|c|c|c|c|c|}
\hline & PDd & PDm & PSd & GMDNDd & GMDNDm & GMDDSd & D160d & $\mathrm{D} 160 \mathrm{~m}$ & D240d \\
\hline PDd & 0,43 & & & & & & & & \\
\hline PDm & 0,55 & 0,20 & & & & & & & \\
\hline PSd & 0,50 & 0,41 & 0,27 & & & & & & \\
\hline GMDNDd & 0,20 & $-0,05$ & 0,09 & 0,45 & & & & & \\
\hline GMDNDm & $-0,19$ & 0,05 & $-0,08$ & 0,45 & 0,28 & & & & \\
\hline GMDDSd & 0,08 & $-0,04$ & 0,78 & 0,03 & $-0,01$ & 0,20 & & & \\
\hline D160d & 0,21 & $-0,02$ & 0,20 & 0,26 & 0,17 & 0,14 & 0,18 & & \\
\hline D $160 m$ & $-0,03$ & 0,12 & $-0,06$ & 0,11 & 0,31 & 0,15 & 0,33 & 0,22 & \\
\hline D240d & 0,47 & 0,19 & 0,23 & 0,23 & 0,08 & 0,33 & 0,53 & 0,45 & 0,15 \\
\hline
\end{tabular}


PD

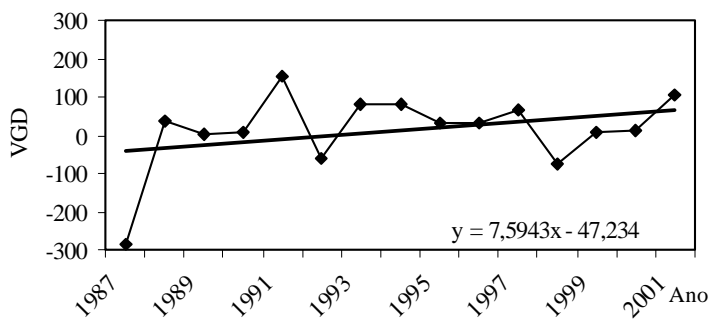

GMDND

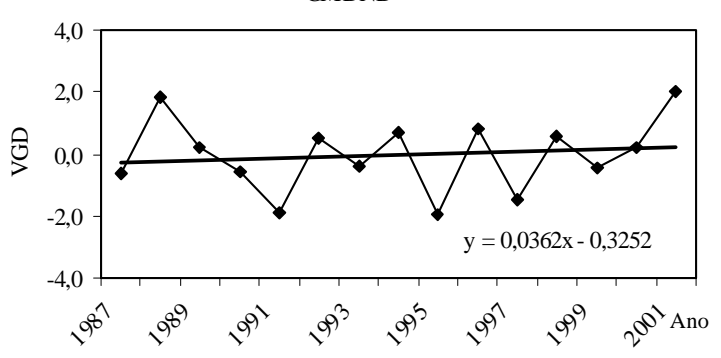

D160

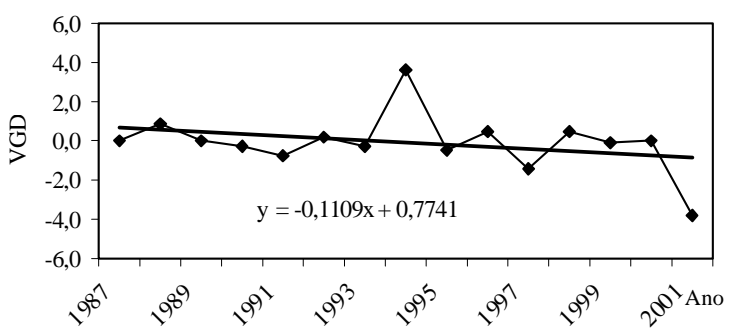

PD

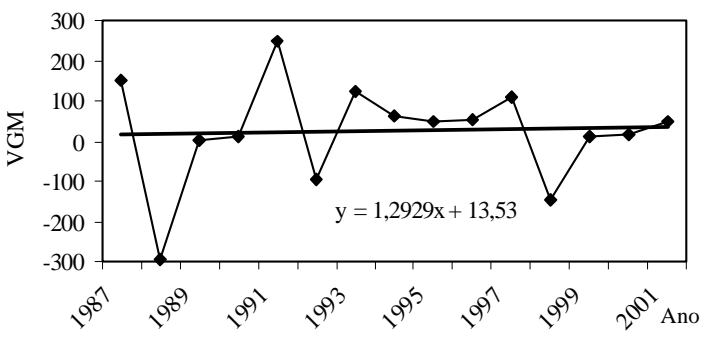

GMDND

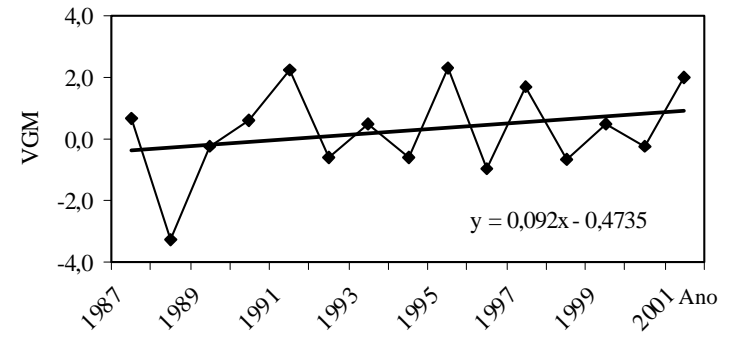

D160

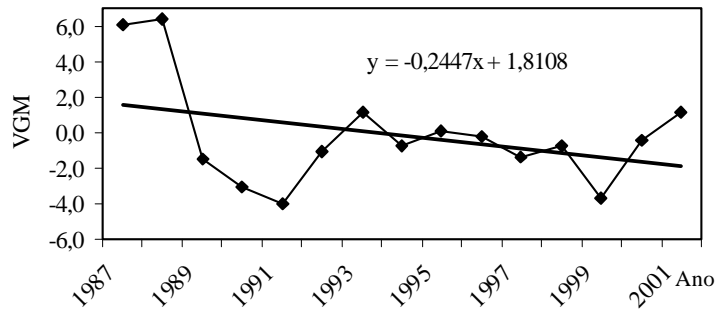

Figura 2 - Valores genéticos diretos (VGD) e maternos (VGM) médios anuais com reta de tendência genética para peso à desmama (PD) e ganho de peso médio diário do nascimento à desmama (GMDND), em gramas, e dias para atingir $160 \mathrm{~kg}$ na pré-desmama (D160), em dias, para uma população da raça Brangus.

presente nas matrizes cruzadas. Resultados semelhantes foram obtidos para o efeito direto por Malhado et al. (2005), que verificaram redução de 0,14 dias/ano para dias para ganhar $160 \mathrm{~kg}$ na fase pré-desmama e, para o materno, estimaram redução próxima de zero para a raça Nelore. Segundo Smith (1985), a taxa de mudança genética anual possível de ser obtida varia de 1 a $3 \%$ da média da população, enquanto neste trabalho os valores foram bastante inferiores.

As tendências genéticas diretas anuais para as características pós-desmama não foram significativas, o que indica que a evolução genética nesta população foi nula no período estudado (Figura 3). Para peso ao sobreano e GMDDS, os valores foram 5,15 e 0,01 g/ano, respectivamente, e estão de acordo com os dados reportados por
Balbé et al. (2007), que relataram valor de -0,029 g/ano para GMDDS em uma população Angus-Nelore, todavia este valor é inferior ao relatado por Holanda et al. (2004) para ganho pós desmama $(75,20 \mathrm{~g} / \mathrm{ano})$ para a raça Nelore. Baixos valores para a tendência genética podem ser explicados, segundo Razook et al. (1993), pela atuação de efeitos ambientais ou pela pequena seleção direcional.

As tendências fenotípicas para as características em estudo (Figuras 4 e 5) foram positivas para todas as características, o que indica que o progresso verificado decorre de melhorias nas condições de ambiente, uma vez que as mudanças genéticas não foram significativas. $O$ valor estimado para GMDDS, de 7,4 g/ano, foi superior ao estimado por Balbé et al. (2007), de 5,69 g/ano para a raça Brangus. 
PS

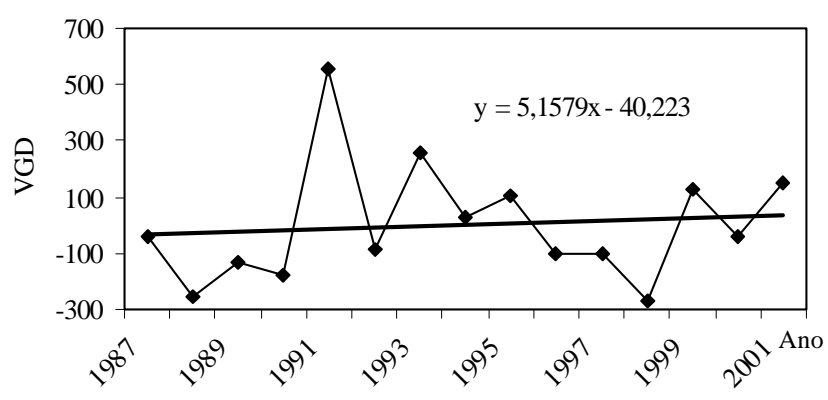

GMDDS

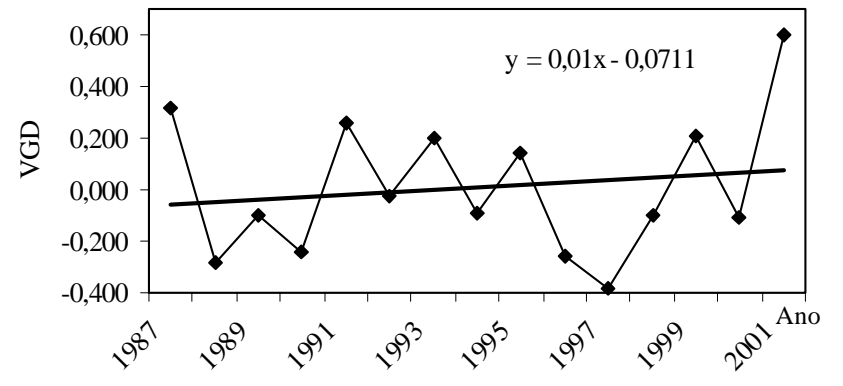

D240

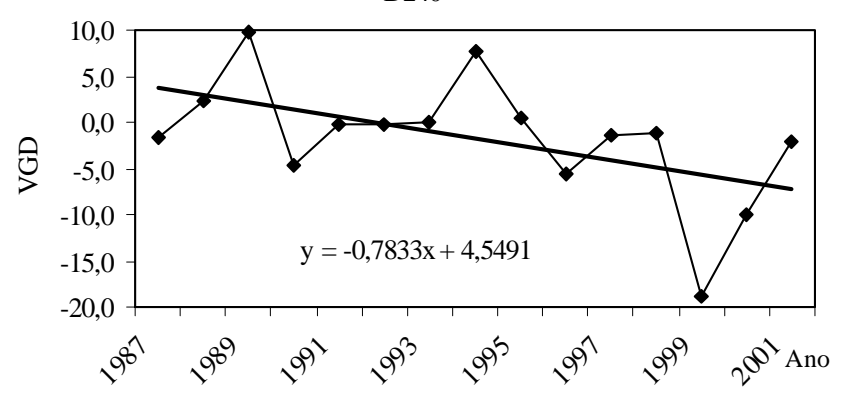

Figura 3 - Valores genéticos diretos (VGD) médios anuais com reta de tendência genética para peso ao sobreano (PS), ganho de peso médio diário ao sobreano (GMDDS), em gramas, e dias para atingir $240 \mathrm{~kg}$ na pós-desmama (D240), em dias, para uma população da raça Brangus.

Resultados indicando baixos progressos fenotípicos são comuns, principalmente quando estimados para rebanhos criados em ambientes distintos, submetidos aos mais diversos sistemas de produção, como é o caso do gado de corte no Brasil, conforme relatado por Silva et al. (1997) e Euclides Filho et al. (2000).
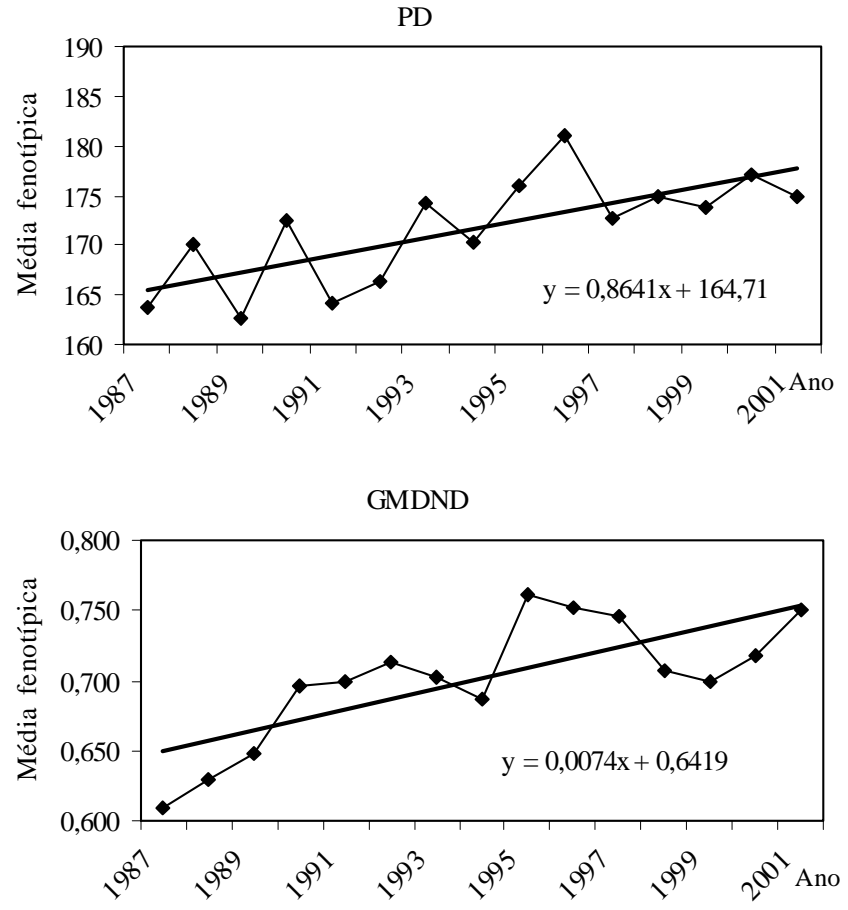

\section{D160}

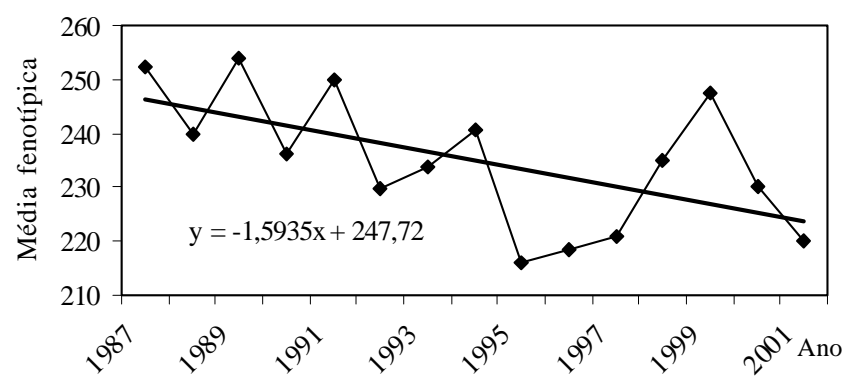

Figura 4 - Médias fenotípicas anuais com reta de tendência fenotípica para peso à desmama (PD), ganho de peso médio diário do nascimento à desmam (GMDND), em quilogramas, e dias para atingir $160 \mathrm{~kg}$ na pré-desmama (D160), em dias, para uma população da raça Brangus.

Considerando que o progresso genético para a população estudada foi muito baixo, embora os produtores estejam conseguindo progresso anual no desempenho com as melhorias nas condições de meio ambiente, se não for promovida melhoria genética nos próximos anos, o progresso fenotípico deverá atingir seu limite. 
PS

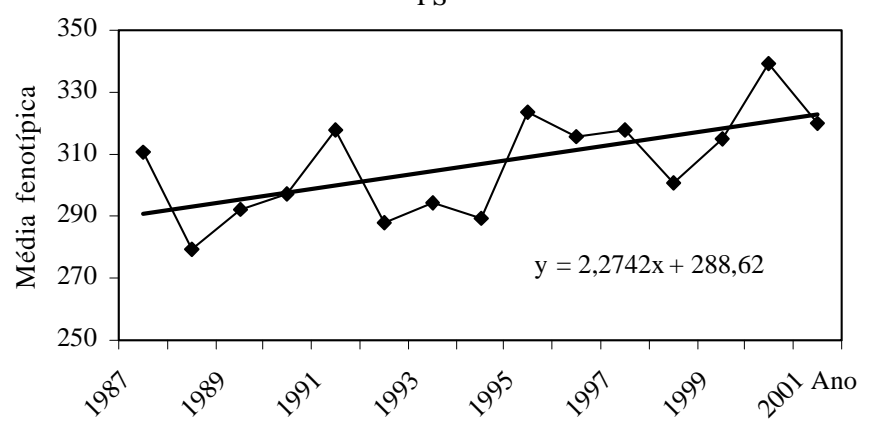

GMDDS

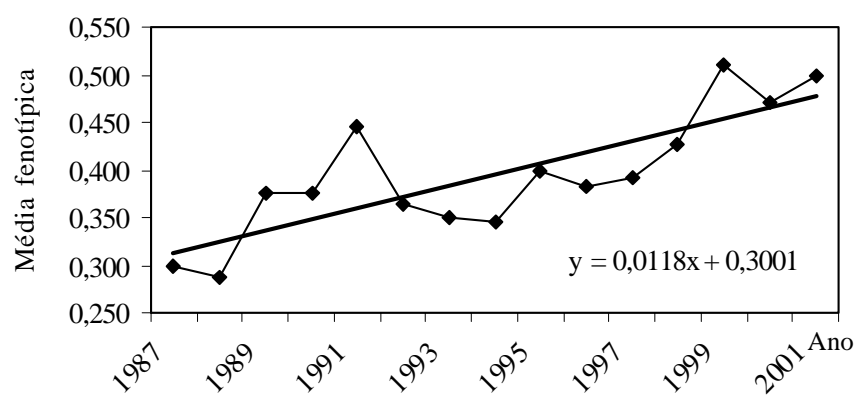

D240

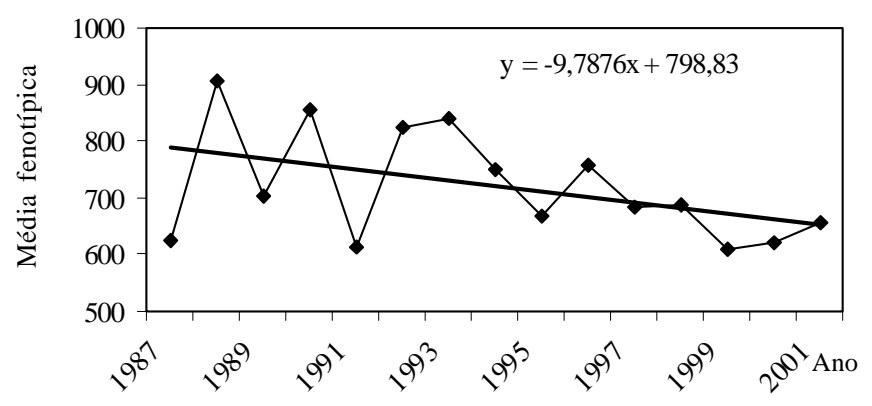

Figura 5 - Médias fenotípicas anuais com reta de tendência fenotípica para peso ao sobreano (PS), ganho de peso médio diário da desmama ao sobreano (GMDDS), em quilogramas, e dias para atingir $240 \mathrm{~kg}$ na pósdesmama (D240), em dias, para uma população da raça Brangus.

\section{Conclusões}

As herdabilidades estimadas para as características peso à desmama e ao sobreano, ganho de peso médio diário do nascimento à desmama e da desmama ao sobreano e dias para ganhar 160 e 240 quilogramas de peso vivo indicam que é possível obter ganho genético por meio de seleção. Os valores genéticos médios, de comportamento pendular, e as tendências genéticas próximas de zero, sugerem não estar ocorrendo progresso genético na popu- lação; no entanto, as tendências fenotípicas positivas indicam que os aumentos anuais obtidos no período resultaram das melhorias nas condições de meio. Portanto, são necessárias medidas que promovam progresso genético, caso contrário, com o passar do tempo, as tendências fenotípicas tenderão a ser nulas.

\section{Agradecimentos}

À Gensys Consultores Associados e Natura Genética Sul-Americana, pela cessão dos arquivos de dados.

\section{Literatura Citada}

BALBÉ, D.D.; RORATO, P.R.N.; ANDREAZZA, J. et al. Tendências genética e fenotípica para ganho de peso médio diário entre a desmama e o sobreano em uma população Angus x Nelore. Arquivo Brasileiro de Medicina Veterinária e Zootecnia, v.59, n.1, p.225-232, 2007.

BOLDMAN, K.H.; KRIESE, L.A.; Van VLECK, L.D. et al. A manual for use for MTDFREML. A set of programs to obtain estimates of variance and covariances (DRAFT). Lincoln: Departament of Agriculture/Agricultural Research Service, 2001. 120p.

ELER, J.P.; VAN VLECK, L.D.; FERRAZ, J.B.S. et al. Estimation of variance due to direct and maternal effects for growth traits of Nelore cattle. Journal of Animal Science, v.73, p.32533258, 1995.

EUCLIDES FILHO, K.; SILVA, L.O.C.; ALVES, R.G.O. et al. Tendência genética da raça Gir. Pesquisa Agropecuária Brasileira, v.35, n.4, p.787-791, 2000.

FERNANDES, H.D.; FERREIRA, G.B.; RORATO, P.N.R. Tendências e parâmetros genéticos para características de crescimento em bovinos Charolês criados no Rio Grande do Sul. Revista Brasileira de Zootecnia, v.31, n.1, p.321-330, 2002.

GARNERO, A.V.; LÔBO, R.B.; BORJAS, A.R. et al. Estimativas de parâmetros genéticos para características incluídas em critérios de seleção em gado de corte. In: REUNIÃO ANUAL DA SOCIEDADE BRASILEIRA DE ZOOTECNIA, 35., 1998, Botucatu. Anais... Botucatu: Sociedade Brasileira de Zootecnia, p.434-436, 1998.

GUTERRES, L.F.W.; RORATO, P.R.N.; BOLIGON, A.A. et al. Efeito da inclusão da covariância genética aditiva direta-materna no modelo de análise sobre a magnitude das estimativas de parâmetros e valores genéticos preditos para ganho de peso na raça Brangus. Ciência Rural, v.37, n.3, p.809-814, 2007.

HILL, W.G. Design of quantitative genetics selection experiments. In: ROBERTSON, A. (Ed.) Selection experiments in laboratory and domestic animals. Slough: CAB, 1980. p.1-13.

HOLANDA, M.C.R.; BARBOSA, S.B.P.; RIBEIRO, A.C. et al. Tendências genéticas para crescimento em bovinos Nelore em Pernambuco, Brasil. Archivos de Zootecnia, v.53, n.202, p.185-194, 2004.

MALHADO, C.H.M.; MARTINS FILHO, R.; LÔBO, R.N.B. et al. Tendências genéticas para características relacionadas à velocidade de crescimento em bovinos Nelore na região Nordeste do Brasil. Revista Brasileira de Zootecnia, v.34, n.1, p.60-65, 2005.

MARCONDES, C.R.; BERGMANN, J.A.G.; ELER, J.P. et al. Análises de alguns critérios de seleção para características de crescimento na raça Nelore. Arquivo Brasileiro de Medicina Veterinária e Zootecnia, v.52, n.1, p.83-89, 2000. 
MARCONDES, C.R.; GAVIO, D.; BITTENCOURT, T.C.C. et al. Estudo de modelo alternativo para estimação de componentes de (co)variância e predição de valores genéticos de características de crescimento em bovinos da raça Nelore. Arquivo Brasileiro de Medicina Veterinária e Zootecnia, v.54, n.1, p.93-99, 2002. MARQUES, L.F.A.; PEREIRA, J.C.C.; OLIVEIRA, H.N. et al. Análise de características de crescimento da raça Simental. Arquivo Brasileiro de Medicina Veterinária e Zootecnia, v.52, n.5, p.527-533, 2000 .

MELLO, S.P.; ALENCAR, M.M.; SILVA, L.O.C. et al. Estimativas de (co)variâncias e tendências genéticas para pesos em um rebanho Canchim. Revista Brasileira de Zootecnia, v.31, n.4, p.1707-1714, 2002.

MERCADANTE, M.E.Z.; LÔBO, R.B. Estimativas de (co)variância e parâmetros genéticos dos efeitos direto e materno de características de crescimento de fêmeas de um rebanho Nelore. Revista Brasileira de Zootecnia, v.26, n.6, p.1124-1133, 1998.

OLIVEIRA, J.A.; LÔBO, R.B.; OLIVEIRA, H.N. et al. Tendência genética em pesos e ganhos em peso de bovinos da raça Guzerá. Pesquisa Agropecuária Brasileira, v.30, n.11, p.1355-1360, 1995.

RAZOOK, A.G.; FIGUEIREDO, L.A.; BONILHA NETO, L.M. et al. Intensidades de seleção e respostas direta e correlacionadas em 10 anos de progênies de bovinos das raças Nelore e Guzerá selecionadas para peso pós-desmame. Boletim da Indústria Animal, v.50, n.2, p.147-163, 1993
SARMENTO, J.L.R.; PIMENTA FILHO, E.C.; RIBEIRO, M.N. et al. Efeitos ambientais e genéticos sobre ganho em peso diário de bovinos Nelore no estado da Paraíba. Revista Brasileira de Zootecnia, v.32, n.2, p.325-330, 2003.

STATISTICAL ANALYSIS SYSTEM - SAS. User's guide. Version 8. Cary: SAS Institute, 2001. (CD-ROM).

SILVA, L.O.C.; EUCLIDES FILHO, K.; NOBRE, P.R.C. et al. Tendência genética na raça Nelore no Brasil. In: REUNIÃO ANUAL DA SOCIEDADE BRASILEIRA DE ZOOTECNIA, 34. 1997, Juiz de Fora. Anais... Juiz de Fora: Sociedade Brasileira de Zootecnia, 1997. p.175.

SMITH, C. Rates of genetic change in farm livestock. Research Development Agriculture, v.1, p.79-85, 1985.

SORENSEN, D.A.; KENNEDY, B.W. Estimation of response to selection using last-squares and mixed model methodology. Journal of Animal Science, v.58, p.1097-1106, 1984.

SORENSEN, D.A.; KENNEDY, B.W. Analysis of selection experiments using mixed model methodology. Journal of Animal Science, v.63, p.245-258, 1986.

SOUZA, J.C.; CAMPOS, L.T.; FREITAS, J.A. et al. Parâmetros genéticos dos pesos ao nascer e aos 205 dias de idade em animais da raça Angus no Brasil. In: REUNIÃO ANUAL DA SOCIEDADE BRASILEIRA DE ZOOTECNIA, 44., 2007, Jaboticabal. Anais... Jaboticabal: Sociedade Brasileira de Zootecnia, 2007. (CD-ROM). 